\title{
Multiple energy scales in mesospin systems: The vertex-frustrated Saint George lattice
}

\author{
Henry Stopfel $\odot,{ }^{1,}{ }^{*}$ Unnar B. Arnalds, ${ }^{2}$ Aaron Stein, ${ }^{3}$ Thomas P. A. Hase $\odot,{ }^{4}$ \\ Björgvin Hjörvarsson, ${ }^{1}$ and Vassilios Kapaklis $\mathbb{1}^{1, \dagger}$ \\ ${ }^{1}$ Department of Physics and Astronomy, Uppsala University, Box 516, SE-75120, Uppsala, Sweden \\ ${ }^{2}$ Science Institute, University of Iceland, Reykjavik, Iceland \\ ${ }^{3}$ Center for Functional Nanomaterials, Brookhaven National Laboratory, Upton, New York 11973, USA \\ ${ }^{4}$ Department of Physics, University of Warwick, Coventry CV4 7AL, United Kingdom
}

(Received 9 July 2021; revised 12 October 2021; accepted 12 November 2021; published 30 November 2021)

\begin{abstract}
The interplay between topology and energy hierarchy plays a vital role in the collective magnetic order in artificial ferroic systems. Here we investigate, experimentally, the effect of having one or two activation energies of interacting Ising-like magnetic islands-mesospins-in thermalized, vertex-frustrated lattices. The thermally arrested magnetic states of the elements were determined using synchrotron-based magnetic microscopy after cooling the samples from temperatures above the Curie temperature of the material. Statistical analysis of the correlations between mesospins across several length scales reveals changes in the magnetic order, reflecting the amount of ground state plaquettes realized for a vertex-frustrated lattice. We show that the latter depends on the presence, or not, of different activation energies.
\end{abstract}

DOI: 10.1103/PhysRevMaterials.5.114410

\section{INTRODUCTION}

Tailoring the field response of magnetic mesospins using a hierarchy of energy scales was originally demonstrated by Cowburn and Welland [1]. In their case, the hierarchy was obtained from a distribution in the size and shape of small magnetic elements which facilitated the design of magnetic cellular automata, with potential uses in information processing and nonvolatile information storage [1-3]. Even though these structures were thermally inactive, their results highlighted the importance of the hierarchy of energies on the observed magnetic order and global response of the system. Since then, the exploration of the magnetic properties of nanoarrays has expanded dramatically [4-6] and now includes arrays designed to exhibit geometrical [7-11] and vertex frustration [12-15]. The pairwise frustration of the interactions between spins at the vertex level is preserved in the kagome geometry $[5,8]$. In lattice architectures based on square artificial spin ice, however, the pairwise frustration is lifted by the different interaction strengths between parallel and perpendicular islands at the vertex level $[7,16,17]$. In recent years, the pairwise frustration for square lattices has been successfully restored by a variety of approaches [9-11]. A different approach was proposed by Morrison et al. [13], creating lattices where it is impossible to choose all vertices to be in their lowest energy configuration, due to topological constraints.

\footnotetext{
*henry.stopfel@physics.uu.se

†vassilios.kapaklis@physics.uu.se
}

Published by the American Physical Society under the terms of the Creative Commons Attribution 4.0 International license. Further distribution of this work must maintain attribution to the author(s) and the published article's title, journal citation, and DOI. Funded by Bibsam.
As a result, these vertex-frustrated structures always contain excited vertices, leading to residual entropy. In addition to tailoring the lattice and geometry of the elements, the materials from which the arrays are fabricated can be chosen to allow thermal fluctuations at suitable temperatures [18]. Such approaches have already enabled the investigation of spontaneous ordering, dynamics, and phase transitions on the mesoscale $[10,19-30]$.

Currently there are only a couple of lattices that have been investigated that incorporate multiple element sizes $[10,12,14,26]$. In Östman et al. [10], small circular elements were placed between the islands within the vertex and used as interaction modifiers to modify the overall energy landscape of the lattice, but their actual magnetic state/orientation was not determined. Even though a difference in activation energy for these two type of mesospins is to be expected, it is not trivial to disentangle the actual contribution of the former, since the dimensionality between the two mesospin types also differs (Ising- and XY-like) [31]. To overcome this shortcoming, systems where activation energies may differ, while maintaining the same mesospin dimensionality, can serve as ideal settings for the detailed study of the multiple energy-scale impact on the derived magnetic order. The first investigations of this kind were performed on the Shakti lattice [26], which until then had not been addressed with respect to the differently sized elements within the lattice $[12,14]$. However, the highly degenerate ground state, together with the high symmetry of the Shakti lattice, masks possible longer-range correlations and ordering, limiting the extent of these earlier studies. Thus, other lattice geometries are needed to probe the effect of energy hierarchy on the ordering within artificial spin ice structures, especially on length scales beyond the nearest neighbor.

In this work we address the effect on the magnetic order of having one or two activation energies influencing the 


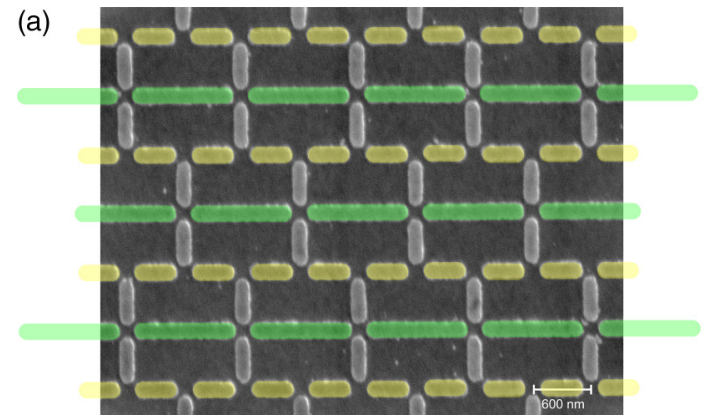

(b)

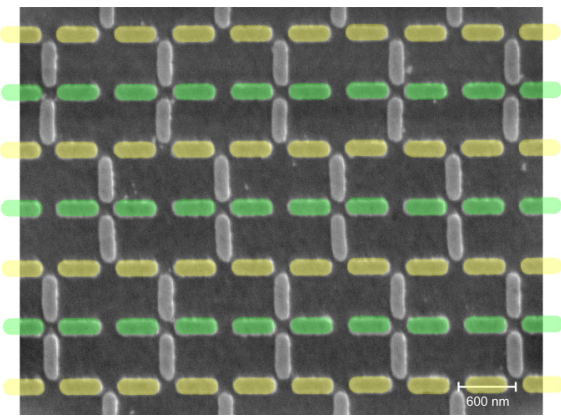

FIG. 1. Illustration of the Saint George lattices. (a) The Saint George (SG) lattice with its two different island (mesospin) sizes. (b) The modified Saint George (mSG) lattice has only one island size. In the Saint George lattice geometry there are two different horizontal chains, colored green and yellow. These horizontal chains are connected by vertical islands. The green island chains are referred to as long-mesospin chains in the main text, as they are consisting of the long islands in the SG lattice and have double the amount of islands in the mSG. The yellow island chains are equal in the SG and $\mathrm{mSG}$ lattice.

magnetic correlations among Ising-like mesospins. We do this experimentally, using mesospins within the Saint George (SG) and modified Saint George (mSG) structures, defined and illustrated in Fig. 1. The SG and $\mathrm{mSG}$ structures are closely related to Shakti lattices described by Chern et al. [12], and constructed in a similar fashion as introduced by Morrison et al. [13]: removing and merging elements, while using the square artificial spin ice lattice as a starting base. As a result, these lattices become vertex frustrated [13], the SG lattice geometry is characterized by its horizontal Ising-like mesospin chains [32,33]. There are two horizontal chain types: one composed of long mesospins and the other with short mesospins. Both chain types are coupled via short vertical mesospins which creates an axial anisotropy in the lattice, as can be seen in Fig. 1. The SG lattice has two activation energies, one for the long and another for the short mesospins. All islands forming the $\mathrm{mSG}$ lattice have the same size and therefore the elements have one and the same intrinsic activation energy.

The ratio of the activation energies within the SG lattice design can give rise to two distinct scenarios: (1) The two energy scales are well separated. This results in a freezing of the long mesospins, while the short mesospins remain thermally active. Lowering the temperature further results in a freezing of the short mesospins, influenced by a static magnetic field originating from the frozen long islands. (2) The energy scales of the short and long mesospins are close or overlapping. This results in an interplay between the differently sized elements during the freezing process. Both scenarios would give rise to an emergent magnetic order, but the correlations between the magnetic mesospins should be different for the two cases. When the activation energies are very different, one may naively expect the long mesospins to behave as an independent subset array, which due to their shape anisotropy and close separation, act as an Ising chain.

\section{METHODS}

\section{A. Sample preparation}

The magnetic nanostructures were fabricated by a postgrowth patterning process on a thin film of $\delta$-doped palladium (iron) [34]. $\delta$-doped palladium (iron) is in fact a trilayer of palladium $(\mathrm{Pd})$ - iron $(\mathrm{Fe})$ - palladium $(\mathrm{Pd})$, where the Curie-temperature $T_{\mathrm{C}}$ and the thermally active temperature range for mesospins is defined by the thickness of the iron layer and their size [26,35]. In the present study the nominal Fe thickness is 2.0 monolayers, characterized by a Curie temperature of $T_{\mathrm{C}}=400 \mathrm{~K}[34,36]$ for the continuous films. The post-patterning was carried out at the Center for Functional Nanomaterials (CFN), Brookhaven National Laboratory in Upton, New York. All investigated structures for the present study, as well as the investigations reported in Stopfel et al. [26], were fabricated on the same substrate from the same $\delta$-doped $\mathrm{Pd}(\mathrm{Fe})$ film, ensuring identical intrinsic material properties as well as the same thermal history for all the investigated structures while performing magnetic imaging. The lengths of the stadium shaped short and long mesospins were 450 and $1050 \mathrm{~nm}$, respectively, while the width was kept at $150 \mathrm{~nm}$ for both. The lattice spacing between two parallel neighboring short elements was chosen to be $600 \mathrm{~nm}$. For more details on the patterning process, see Stopfel et al. [26].

\section{B. Thermal protocol}

The thermal protocol involves gradual cooling from a superparamagnetic state of the patterned elements, to an arrested state at the lowest temperatures. Similar to previous studies on the Shakti lattice [26] and unlike the investigations on square artificial spin ice $[20,23]$ the SG lattice exhibits two distinctive thermally active regimes, caused by the different sizes of the elements. Below the Curie temperature $\left(T_{\mathrm{C}}\right)$ the elements are magnetic and considered as mesospins. The temperature-dependent magnetostatic coupling influences the activation of the elements and is therefore biasing one of the two magnetization directions, depending on the states of the adjacent mesospins.

\section{Determination of the magnetization direction}

The magnetic state of each mesospin was determined by photoemission electron microscopy (PEEM) using the x-ray magnetic circular dichroism (XMCD) contrast. The experimental studies were performed at the 11.0.1 PEEM3 beamline of the Advanced Light Source, in Berkeley, California, USA. The islands were oriented $45 \mathrm{deg}$ with respect to the incoming $\mathrm{X}$-ray beam. Multiple PEEM-XMCD images were acquired 
(a)

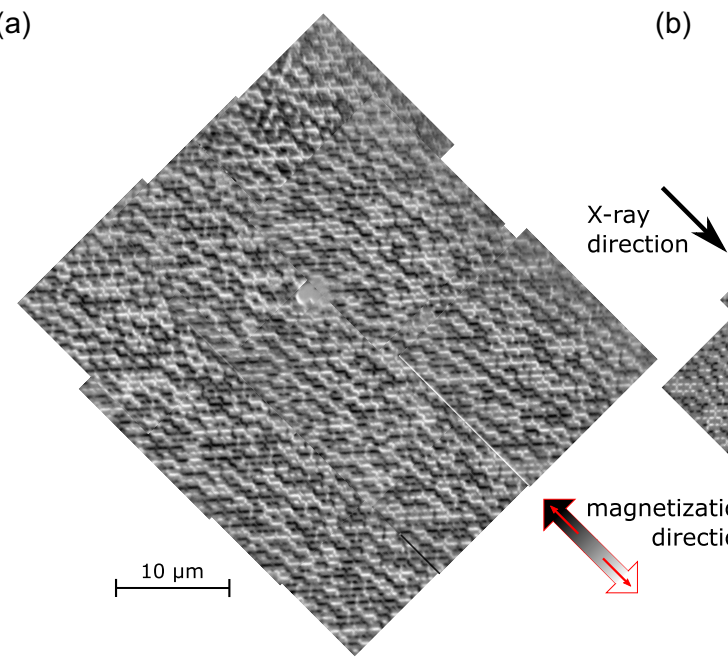

(b)

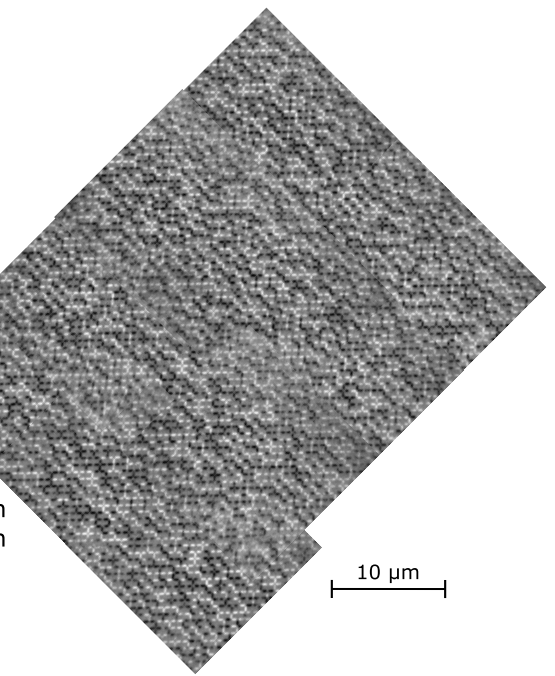

FIG. 2. Photoemission electron microscopy images using the x-ray magnetic circular dichroism effect to visualize the magnetization direction. Combined measurements of (a) the SG lattice and (b) of the mSG lattice. The magnetization direction of each individual island can be determined by its color. Black illustrated mesospins pointing left or up while white mesospins have their magnetization pointing right or down.

and stitched together to form an extended image of more than 5000 mesospins. The PEEM-XMCD images were taken at $65 \mathrm{~K}$, far below the Curie temperature and the blocking temperatures of the mesospins. The PEEM-XMCD images were obtained with a sampling time of $t_{s}=360 \mathrm{~s}$, which defines the time resolution of the measurements: At temperatures far below the blocking temperature of the elements, all magnetic states are stable (frozen) and can be imaged for long periods of time. On the other extreme (higher temperatures), when the magnetization reversal times of the mesospins are much smaller than $t_{s}$ and the elements change their magnetic orientation during the duration of the measurement, no magnetic contrast is obtained.

\section{Activation energies}

The intrinsic activation energies of the mesospins are known from earlier investigations, see Stopfel et al. [26]: $E_{\text {short }} / k_{B}=560(40) \mathrm{K}$ and $E_{\text {long }} / k_{B}=1140(140) \mathrm{K}$. The ratio of the activation energies of noninteracting short and long mesospins is therefore 0.49(4). The Shakti lattices from Stopfel et al. [26] and the ones discussed here were fabricated on the same chip and measured during the same beamtime at PEEM3, removing any uncertainty concerning the intrinsic properties of the material. The ratio of the activation energies of the short and the long mesospins represents a substantial difference and therefore motivates our investigation of the influence of the energy hierarchy on the magnetic ordering mechanism in such multiple energy-scale systems.

\section{RESULTS AND DISCUSSIONS}

The results from photoemission electron microscopy (PEEM-XMCD) experiments for both the SG and $\mathrm{mSG}$ lattice are illustrated in Fig. 2. While the magnetization direction of each mesospin can be determined, it is difficult to identify any correlations in and/or between the SG and mSG lattices from a visual inspection of Fig. 2. To explore the possible effect of different energy hierarchies requires, therefore, a statistical analysis, as described below.

\section{A. Long-mesospin chains}

In Fig. 3 we show the correlation between the magnetization direction of the neighboring long islands, after cooling the sample from room temperature to $65 \mathrm{~K}$. The correlation in the magnetization direction of neighboring islands is calculated using

$$
C^{n}=\frac{\sum_{k}^{N} m_{k} m_{n_{k}}}{N}
$$

Here $N$ is the total number of long mesospins, $m_{k}$ is the magnetization direction of the reference mesospin, and $m_{n_{k}}$ is the magnetization direction of the $n$th neighbor of mesospin $k$. In this formalism, $m_{k}$ and $m_{n_{k}}$ can be \pm 1 depending on their magnetization direction (black or white in Fig. 2). To determine the correlations in the mSG lattice, the same approach using Eq. (1) was applied, but due to the different local starting condition [identified in Fig. 3(b) with $\star$ or + ] the correlations were determined separately for each condition. For the long mesospins, the correlation of the first neighbor is close to be zero, indicating a random arrangement of the long mesospins. This is in contrast to the ferromagnetic order which would have been naively expected [33], calling for an analysis of the vertex states.

\section{B. Vertex abundances}

Statistical analysis of the vertex configurations of the mesospins allows for some insight into the role of the interactions and the degree of order in our arrays. The vertex types, illustrated on the right side of Fig. 4, have different degeneracies: type $\mathrm{I}_{4}$ has a degeneracy of 2 , while type $\mathrm{II}_{4}$, type $\mathrm{III}_{4}$, and type $\mathrm{IV}_{4}$ have a degeneracy of 4,8 , and 2, respectively. To illustrate the difference from a random distribution, we 

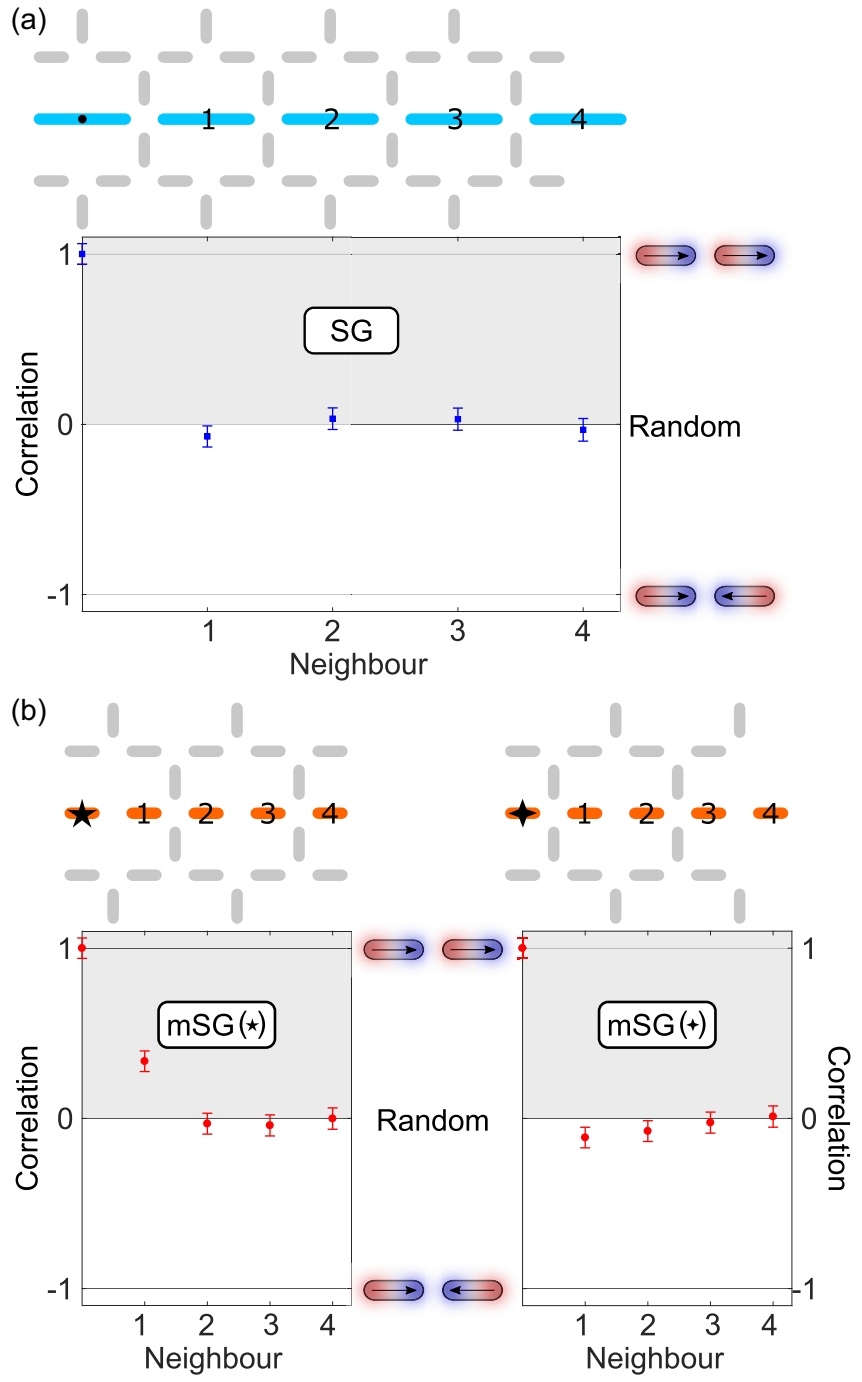

FIG. 3. Magnetic order of long-mesospin chains in the SG and mSG lattice. (a) Correlation between neighboring long mesospins in the SG lattice shows an almost random alignment for the first mesospin neighbor, the slight negative correlation value indicates a preference towards antiferromagnetic order. (b) The same mesospin chains are also investigated in the $\mathrm{mSG}$, where two short mesospins resemble one long mesospin. Depending on the starting element ( $\star$ or + ), the first mesospin neighbor has a positive value $(\star)$ and therefore shows a preference for ferromagnetic alignment, or a negative value $(+)$ and therefore a slight preference for antiferromagnetic alignment.

show the degeneracy corrected abundance for the fourfold and threefold coordinated vertices. To this end, we divide the vertex counts by their degeneracy, normalizing these new degeneracy-corrected vertex counts to one. The degeneracies for the threefold coordinated vertices are 2, 4, and 2 for type $\mathrm{I}_{3}$, type $\mathrm{II}_{3}$, and type $\mathrm{III}_{3}$, respectively. The larger number of low energy vertex configurations as seen in Fig. 4 clearly shows that the mesospins are interacting in both SG and $\mathrm{mSG}$ lattices. However, the number of higher excitations in both the fourfold and threefold vertices are a manifestation of thermal activity during the freezing process. These high energy vertex states (type $\mathrm{II}_{4}-\mathrm{IV}_{4}$ and type $\mathrm{II}_{3}-\mathrm{III}_{3}$, see Fig. 4 for vertex
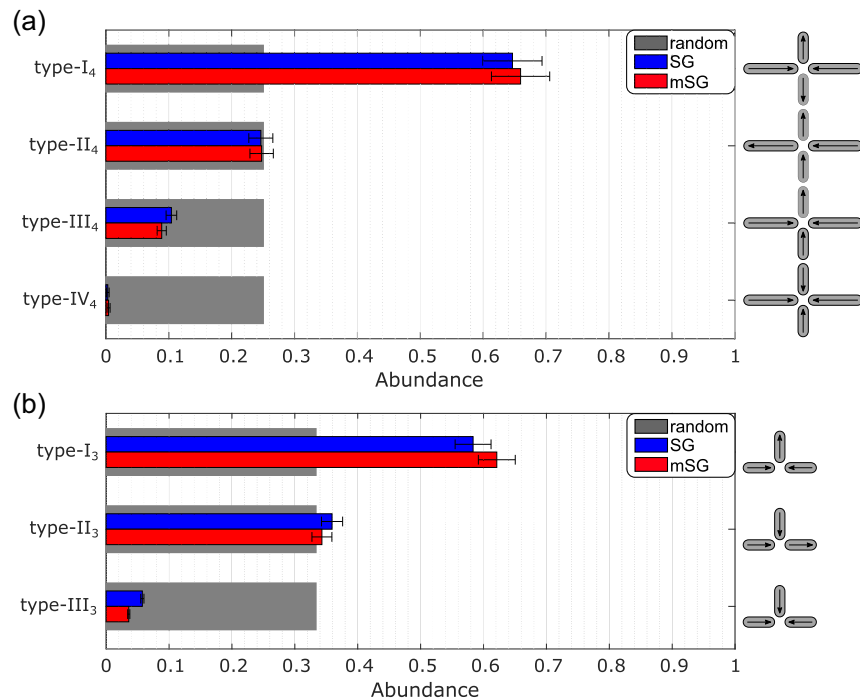

FIG. 4. Vertex statistics for the SG and mSG lattice. (a) The degeneracy corrected vertex abundance for the fourfold coordinated vertices in the SG (blue) and $\mathrm{mSG}$ (red) lattice. (b) The degeneracy corrected vertex abundance for the threefold coordinated vertices in the SG (blue) and mSG (red) lattice. The gray bars illustrate the vertex abundance for a random distribution as it would be received if the mesospins would not interact. The vertex type energies are corresponding to their notation, namely type $\mathrm{I}_{4(3)}$ being the lowest energy and therefore the ground state for the fourfold (threefold) coordinated vertex.

type illustration) have been observed earlier using the same magnetic material [26]. While the distribution of vertex states are not random, it is still not possible to identify significant differences between the SG and mSG lattices. Therefore, we conclude that on the length scale of the fourfold and threefold coordinated vertices, no significant difference between the SG and $\mathrm{mSG}$ lattice can be identified, along with no measurable influence of the different energy scales on the magnetic ordering. So far we have investigated the correlations of the magnetization direction of neighboring mesospins as well as the correlations of three and four interacting mesospins at a vertex. The next length scale at which the anisotropy impacts the emergent behavior is, therefore, across several interacting mesospins and vertices.

\section{Flux closure loops}

Flux closure loops are a way to minimize the energy of the lattice by reducing the residual stray fields. The flux closure loops in the SG and mSG lattice are defined as follows: The smallest flux closure loop in the SG lattice consists of one long and four short mesospins and in the mSG of six short mesospins, as depicted graphically in Fig. 5. The fully flux closed loops are defined as having a circulation +1 for a clockwise and -1 for an anticlockwise flux closure. Energetically, the most favorable circulations will be the fully closed loops with a circulation of \pm 1 . For higher energy states, where one or more islands are reversed, the normalized circulation is modified by \pm 0.4 for each mesospin flipped in the SG lattice and by $\pm \frac{1}{3}$ in the mSG lattice. In Fig. 5 we compare the abundance of full and partial flux closure for the SG and the 

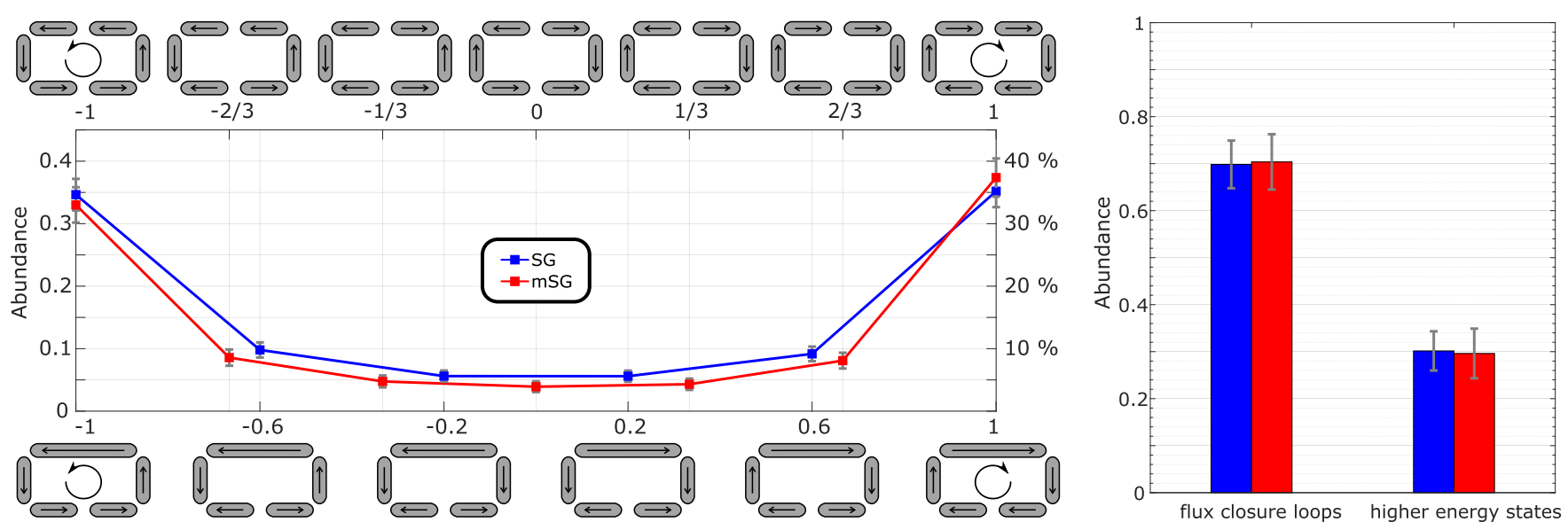

FIG. 5. Mesospin circulation in the SG and mSG lattice. The circulation for loops of five mesospin in the SG lattice (blue), compared to the circulation for six-mesospin loops in the mSG lattice (red) are shown in the left graph. The circulation of the loops was normalized to $+1(-1)$ for a total clockwise (anticlockwise) alignment of all mesospins of a loop. This normalization results in a change of the loop circulation by \pm 0.4 for each mesospin flipped in the SG lattice and by $\pm \frac{1}{3}$ in the $\mathrm{mSG}$ lattice. The right graph illustrates the degeneracy corrected abundance of flux closure loops and higher energy states for both the SG and mSG.

mSG lattices. The low energy flux closure loops, with values of \pm 1 , have the same abundance in the SG and the $\mathrm{mSG}$ lattice. The higher energy states, with one or more reversed mesospins at first glance, appear more abundant in the SG lattice, but combining all flux closure loops (circulation values \pm 1 ) to one value and summing up all remaining higher energy states to another value, it becomes clear that there are no significant differences between the flux closure loops in the SG and the mSG lattice (right graph in Fig 5). Judging from this loop circulation evaluation in Fig. 5 and the vertex statistics in Fig. 4, we can assume that the SG and $\mathrm{mSG}$ lattice are similarly ordered and there is no major differences between them, originating from the different energy scales. Thus far we have only observed ordering and correlation at the shortrange level, such as the vertex configurations and the smallest possible flux closure loops. To identify the next length scale, we now turn our attention to the collective ground state of the SG lattice.

\section{Ground state manifold}

The analysis presented above, focusing at the short-range scale, clearly indicates that the energy scales of the two sizes overlap and drive an elaborate ordering mechanism. We can simplify the evaluation of the ground state manifold by only minimizing the vertex energies, not taking into account the two different activation energies for the long and short mesospins. This results in the ground state manifold which we present in Fig. 6 for one of the possible ground state configurations for the SG and the mSG lattice.

All fourfold coordinated vertices in the SG ground state manifold are in their energetically lowest state, the type- $\mathrm{I}_{4}$
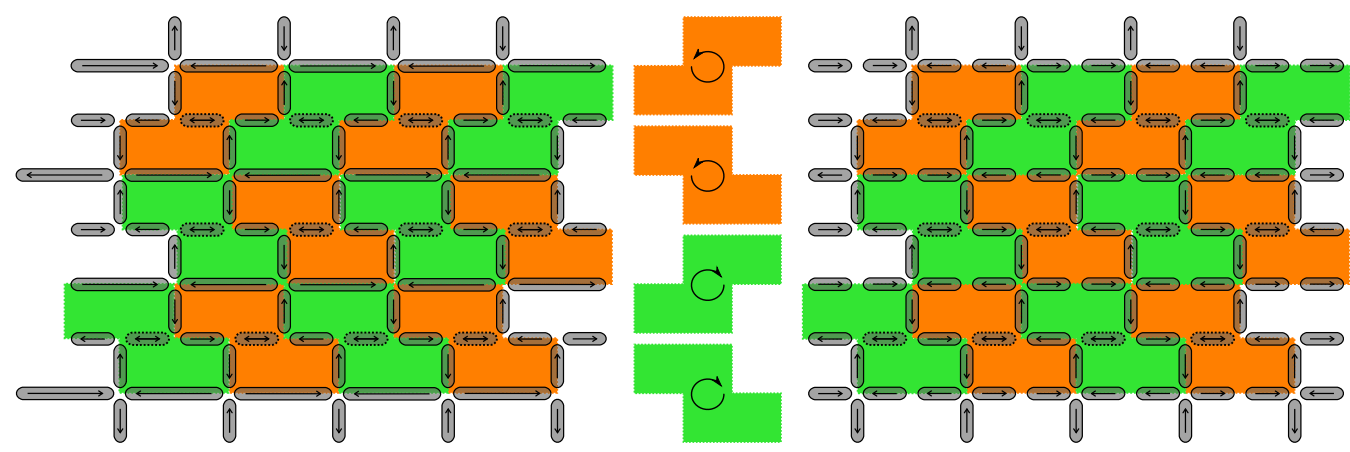

FIG. 6. Proposed ground state configuration in the SG (left) and mSG lattice (right). This ground state configuration takes into account only the vertex energies, while ignoring the different activation energies for short and long mesospins. All fourfold-coordinated vertices are in their lowest energy state. This configuration leads to a frustration among the threefold-coordinated vertices, as $50 \%$ of them are in their lowest energy state but the other 50\% are in their first excited state, equivalent to the Shakti lattice [12]. The proposed ground state creates flux closure loops, as unit cells of the ground state manifold. The four different flux closure loops are colored orange or green, depending on their sense of flux circulation (sense of rotation). The border mesospins, eight in the SG and ten in the mSG lattice, are head to tail aligned, with an either clockwise or anticlockwise sense of rotation. The nonborder mesospin is frustrated and located in the center of the flux closure loop. The magnetic orientation of this island has no impact on the overall energy of the entire lattice, but it defines the position of the excited threefold-coordinated vertex. 

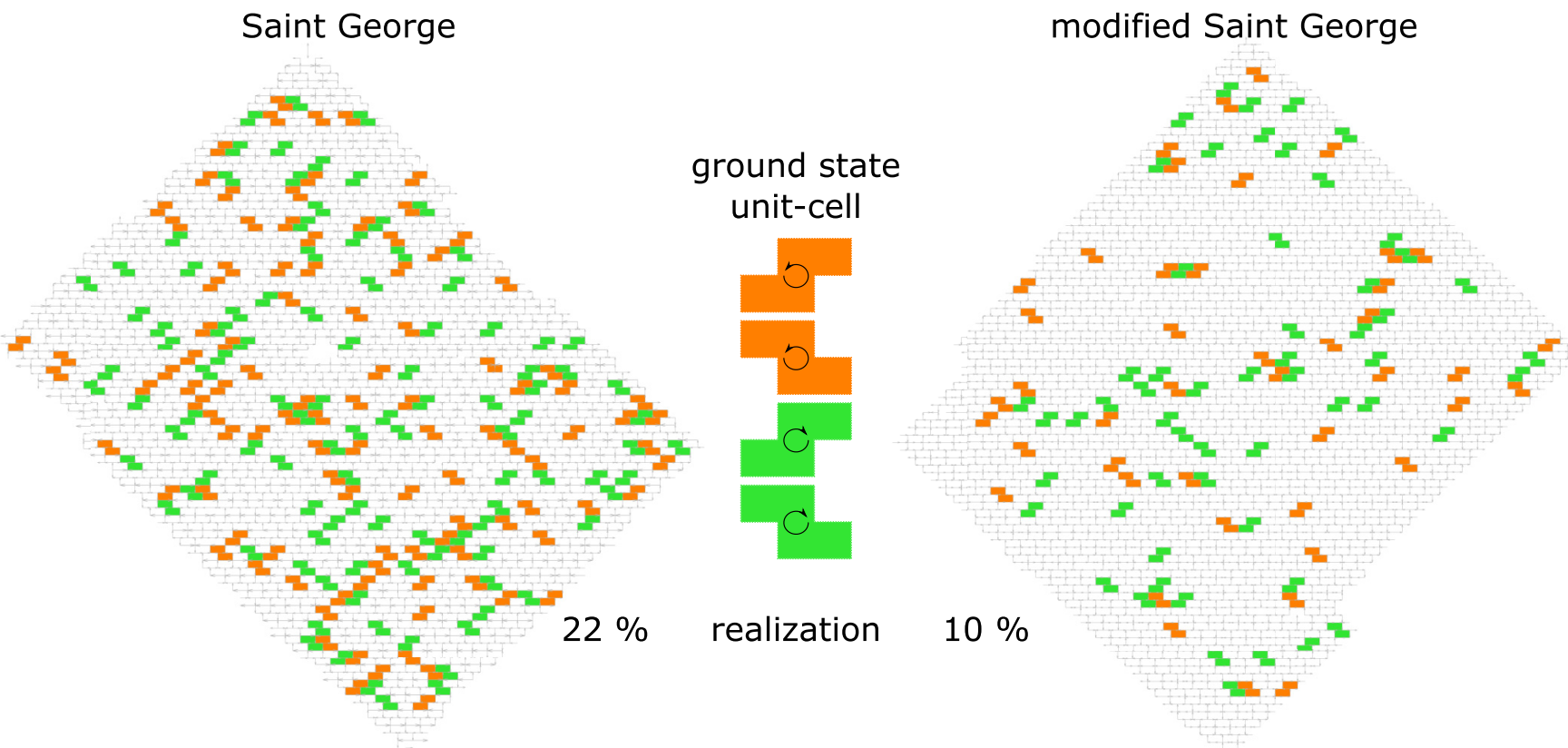

FIG. 7. Mapping of ground state unit cells in the SG and mSG lattice. The ground state unit cells introduced in Fig. 6 are mapped out experimentally in our arrays. There is a clear difference in the quantity of ground state unit cells found in the SG and the mSG lattice. A $22 \%$ ground state realization is observed in the SG lattice, with a $10 \%$ realization in the $\mathrm{mSG}$ lattice.

state (see Fig. 4 for state notations). The geometrical configuration of the SG lattice leads to a vertex frustration at the threefold coordinated vertices, which is present at all temperatures. This vertex frustration results in $50 \%$ of all threefold coordinated vertices being in their lowest energy state (type $\mathrm{I}_{3}$ ), with the remaining $50 \%$ in their first excited state (type $\mathrm{II}_{3}$ ). The $\mathrm{SG}$ lattice geometry also yields a high degree of degeneracy, as the arrangement of the ground state unit cells (illustrated in Fig. 6) can be achieved in numerous ways. In addition, the central mesospin, inside these ground state unit cells, defines the position of the type- $\mathrm{II}_{3}$ excitation and is energetically degenerate. In a real sample, in which the dynamics of the freezing process itself also play a role, excitations and localized disorder are inevitably locked into the system which prevents long-range ground states from forming. This has been observed in the vertex statistics presented for these (Fig. 4) and similar artificial arrays such as the Shakti lattice [26].

All of the analyses until now took place in the short range and among two, three, four, five, or six mesospins, residing in the short-range order. Looking in more detail at the ground state unit cell, it becomes evident that flux closure loops consisting of two long and six short mesospins for the SG lattice and ten short mesospins for the mSG lattice should also be considered when discussing the overall ordering of the lattice geometry. Furthermore, these ground state unit cells with their eight or ten mesospins represent an intermediate length scale within the SG lattice. As the ground state unit cells can be arranged in multiple ways throughout the lattice geometry, the flux closure loops cannot easily be investigated in the same fashion as we did with the circulation values in Fig. 5. It is possible however, to investigate and compare directly their observed abundances in the lattices.

\section{E. Magnetic ordering on an intermediate length scale}

In pursuit of this magnetic ordering on an intermediate length scale, we turn our attention towards the ground state unit cells in our measured arrays (Fig. 2). In this way we are able to detect the first major differences between the SG and the $\mathrm{mSG}$ lattices by counting the number of ground state unit cells present in the arrays. Figure 7 illustrates the distribution of these ground state unit cells in the measured lattices and in this representation the fact that more ground state unit cells can be found in the SG lattice, than in the mSG lattice, is clearly highlighted. Evaluating further these ground state unit cells quantitatively, we find $22 \%$ ground state realization for the SG lattice but only $10 \%$ ground state realization for the $\mathrm{mSG}$ lattice. This difference is attributed to the interplay of the two energy scales, where the long mesopins influence the ordering of the short mesospins on length scales beyond that of nearest neighbors. The highly susceptible short-mesospin matrix acts as an interaction enhancer and propagates the influence of the long mesospins into the intermediate length scale, which is reflected in the result presented in Fig. 7 and related ground state abundances. Accounting for the amount of elements present in a ground state unit cell one can argue that the retrieved values are just a reflection of the degrees of freedom, which are reduced in the SG lattice when compared with the mSG lattice.

\section{F. Conditional mesospin arrangement}

We have also investigated a conditional arrangement of mesospins wherein the two short mesospins representing the long mesospin in the $\mathrm{mSG}$ lattice have to be ferromagnetically aligned to each other (see Fig. 8). As a first step we identify two long mesospins in two subsequent long-mesospin chains in the SG lattice (red mesospins in Fig. 8). These mesospins 


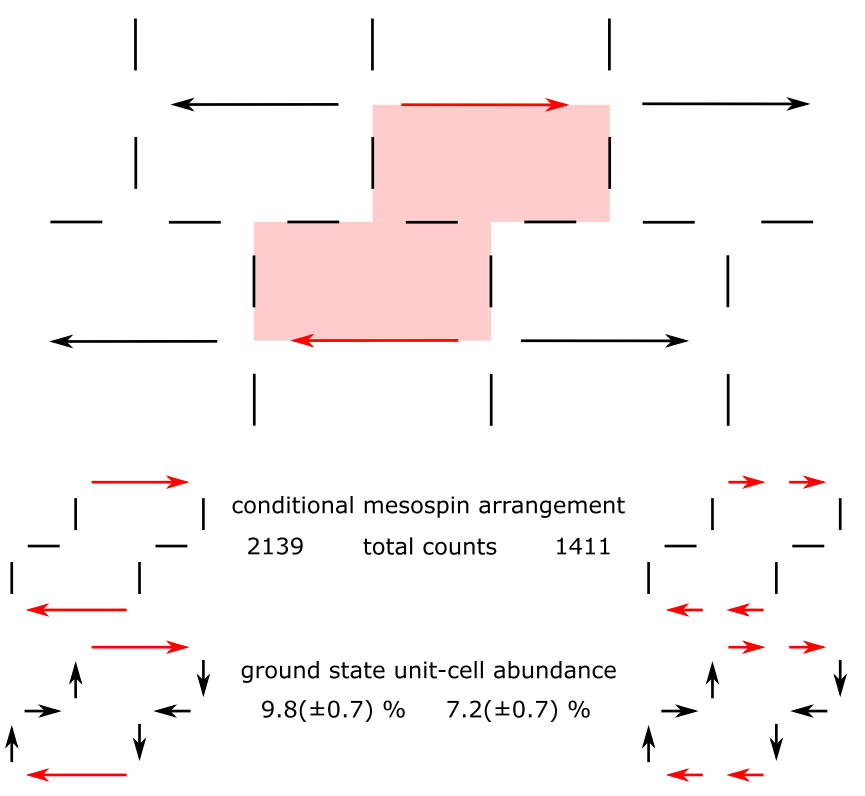

FIG. 8. Conditional arrangement of the ground state unit cells in the SG and mSG lattice. The two long mesospins from subsequent long-mesospin chains are antiferromagnetically aligned to each other. In this conditional mesospin arrangement we compare the abundance of the ground state unit cells in the SG and $\mathrm{mSG}$ lattice and see a clear evidence of a stronger ordering in the SG lattice.

must have an antiparallel alignment of their magnetization direction ignoring the orientation of the six short mesospins connecting the two. In order to have the same predefined condition in the $\mathrm{mSG}$ lattice, we perform the same selection, but this time we additionally choose that the two short mesospins (corresponding to the long mesospin in the SG lattice) being ferromagnetically aligned to each other while the magnetization direction in the subsequent chains is still antiparallel oriented. The magnetization of the long mesospins (or two short mesospins in the $\mathrm{mSG}$ ) in the subsequent mesospin chains is set in the conditional arrangement and we focus our attention to the subset of the six short mesospins connecting the selected mesospin configuration. In this way we restrict the degrees of freedom in this subset of mesospins in the $\mathrm{mSG}$ to be the same as that in the SG lattice. Searching for arrangements where two long mesospins of subsequent longisland chains are antiferromagnetically aligned to each other, we observed a total of 2139 and 1411 instances in the SG and $\mathrm{mSG}$ lattices, respectively. The different abundance for this conditional arrangement in the SG and mSG demonstrating the influence of the degrees of freedom. By investigating these conditional arrangements for the ground state unit cells we are comparing a subset of mesospins (six short mesospins), which is identical in the $\mathrm{SG}$ and $\mathrm{mSG}$ lattice. If these six mesospins are randomly arranged we would receive an abundance of $\frac{1}{2^{6}}=1.6 \%$. Investigating the conditional arrangements for ground state unit cells an abundance of $9.8( \pm 0.7) \%$ for the SG and $7.2( \pm 0.7) \%$ for the $\mathrm{mSG}$ can be found. These values are clearly distinguishable from a random arrangement, considering their uncertainty and can therefore be attributed to the interaction energies in these lattices. It is striking to see a difference between the SG and mSG lattice only at this length scale. The different abundance of ground state unit cells in the conditional mesospin arrangements can only be explained by the difference in activation energy for the short and long mesospins. The conditional mesospin arrangement shows therefore the direct influence of this activation energy on the intermediate-range ordering in mesoscopic spin systems.

\section{CONCLUSION}

The analysis of the results of this study indicate that the long mesospins act as ordering seeds, around which the short mesospins preferably align themselves, which is in agreement with previous studies of the Shakti lattice [26]. In contrast to the latter case though, here the short mesospins affect the character of the magnetic order among the long mesospins, through an apparently stronger interaction between the two distinct mesospin energy scales, arising from the lattice geometry. These fluctuating Ising-like short mesospins can be seen as interaction modifiers for the long-mesospin chains, in a similar way as recently presented by Östman et al. [10], where the placement of magnetic disks in the center of the square artificial spin ice vertex alters the strength and the degeneracy of the magnetic coupling between the Ising-like mesospins. In the case of the SG lattice, the short mesospins are placed in between the long mesospins but with a horizontal offset. During the cooling process the long mesospins strongly interact with the highly susceptible fluctuating short-mesospin matrix, modifying the normally ferromagnetic correlations between the long mesospins. As such, the interplay between the energy hierarchy and the topology needs to be considered, as the resulting magnetic order cannot be understood by simply following a strict separation between the short- and longmesospin ordering. A domination of the energy hierarchy over the topological influence would favor predominantly the formation of ferromagnetic chains along the long-mesospin chains, which we do not observe in these samples. A detailed investigation of the exact ordering mechanism during the freezing process in such systems, as for example while varying the cooling rate, would shed more light on the interplay between the different energy scales.

Focusing on the short-range order, we observed that there are no significant differences between the SG and the mSG lattice, neither at the vertex abundances nor the smallest flux closure loops length scale. These observations hint that the two energy scales have no impact on the magnetic ordering, but turning our attention to the intermediate length scale we see a significant lower ground state realization in the $\mathrm{mSG}$ lattice, which is independent of the degrees of freedom. We therefore conclude that the two step freezing process in the SG lattice allows the mesospin system to achieve twice as much ground state realization as in the one step freezing system of the $\mathrm{mSG}$ lattice. This can be explained as an intermediaterange ordering originating from the long mesospins, while the short-range order is dominated by the short mesospins. The intermediate length-scale order is improved in the SG lattice by the long mesospins interacting with the short-mesospin matrix during the freezing process, which is finally expressed in the higher degree of order on this length scale. While the short-range order is purely dominated by the activation 
energy of the short mesospins, reflected in nondistinguishable magnetic ordering in the SG and $\mathrm{mSG}$ at the short range.

Our study highlights the importance of the energy scales in combination with the topology on the collective magnetic order in artificial ferroic systems. A full understanding of this interplay between energy scales and topology, makes it possible to use artificial magnetic nanostructures with differently sized [26] and shaped elements [10,35], in order to design systems where emergence and frustration can be studied in a systematic way at the mesoscale [5,37]. Furthermore, this work calls for the development and studies of appropriate models accounting for the presence of multiple energy scales [38], in settings where geometry and/or topology have a significance. This approach may have also long-term importance for the design of arrays with enhanced magnetic reconfigurability, which can be utilized for instance in magnonics [39-42], exploiting the dependence of their dynamic magnetization spectra on their micromagnetic states $[18,43,44]$.

\section{ACKNOWLEDGMENTS}

The authors acknowledge support from the Knut and Alice Wallenberg Foundation project "Harnessing light and spins through plasmons at the nanoscale" (2015.0060), the Swedish Foundation for International Cooperation in Research and Higher Education (Project No. KO2016-6889), and the Swedish Research Council (Project No. 2019-03581). The patterning was performed at the Center for Functional Nanomaterials, Brookhaven National Laboratory, supported by the U.S. Department of Energy, Office of Basic Energy Sciences, under Contract No. DE-SC0012704. This research used resources of the Advanced Light Source, which is a DOE Office of Science User Facility under contract No. DE-AC02-05CH11231. U.B.A. acknowledges funding from the Icelandic Research Fund Grants No. 207111 and No. 152483 and the University of Iceland Research Fund. The authors would also like to express their gratitude to Dr. Erik Östman for assistance with PEEM-XMCD measurements and valuable conversations, Dr. David Greving for help with the electron-beam lithography, as well as to Dr. Cristiano Nisoli (Los Alamos National Laboratory, USA) and Dr. Ioan-Augustin Chioar (Yale University, USA) for fruitful discussions and valuable feedback. The excellent on-site support by Dr. Andreas Scholl and Dr. Rajesh V. Chopdekar, at the 11.0.1 PEEM3 beamline of the Advanced LightSource, in Berkeley, California, USA, is also greatly acknowledged.
[1] R. P. Cowburn and M. Welland, Science 287, 1466 (2000).

[2] R. Cowburn, A. Adeyeye, and M. Welland, New J. Phys. 1, 16 (1999).

[3] A. Imre, G. Csaba, L. Ji, A. Orlov, G. H. Bernstein, and W. Porod, Science 311, 205 (2006).

[4] L. J. Heyderman and R. L. Stamps, J. Phys.: Condens. Matter 25, 363201 (2013).

[5] C. Nisoli, V. Kapaklis, and P. Schiffer, Nat. Phys. 13, 200 (2017).

[6] N. Rougemaille and B. Canals, Eur. Phys. J. B 92, 62 (2019).

[7] R. F. Wang, C. Nisoli, R. S. Freitas, J. Li, W. McConville, B. J. Cooley, M. S. Lund, N. Samarth, C. Leighton, V. H. Crespi, and P. Schiffer, Nature (London) 439, 303 (2006).

[8] B. Canals, I.-A. Chioar, V.-D. Nguyen, M. Hehn, D. Lacour, F. Montaigne, A. Locatelli, T. O. Menteş, B. S. Burgos, and N. Rougemaille, Nat. Commun. 7, 11446 (2016).

[9] Y. Perrin, B. Canals, and N. Rougemaille, Nature (London) 540, 410 (2016).

[10] E. Östman, H. Stopfel, I.-A. Chioar, U. B. Arnalds, A. Stein, V. Kapaklis, and B. Hjörvarsson, Nat. Phys. 14, 375 (2018).

[11] A. Farhan, M. Saccone, C. F. Petersen, S. Dhuey, R. V. Chopdekar, Y.-L. Huang, N. Kent, Z. Chen, M. J. Alava, T. Lippert, A. Scholl, and S. van Dijken, Sci. Adv. 5, eaav6380 (2019).

[12] G.-W. Chern, M. J. Morrison, and C. Nisoli, Phys. Rev. Lett. 111, 177201 (2013).

[13] M. J. Morrison, T. R. Nelson, and C. Nisoli, New J. Phys. 15, 045009 (2013).

[14] I. Gilbert, G.-W. Chern, S. Zhang, L. O'Brien, B. Fore, C. Nisoli, and P. Schiffer, Nat. Phys. 10, 670 (2014).

[15] I. Gilbert, Y. Lao, I. Carrasquillo, L. O’Brien, J. D. Watts, M. Manno, C. Leighton, A. Scholl, C. Nisoli, and P. Schiffer, Nat. Phys. 12, 162 (2016).
[16] G. Möller and R. Moessner, Phys. Rev. Lett. 96, 237202 (2006).

[17] D. Thonig, S. Reissaus, I. Mertig, and J. Henk, J. Phys.: Condens. Matter 26, 266006 (2014).

[18] S. D. Slöetjes, B. Hjörvarsson, and V. Kapaklis, Appl. Phys. Lett. 118, 142407 (2021).

[19] J. P. Morgan, A. Stein, S. Langridge, and C. H. Marrows, Nat. Phys. 7, 75 (2010).

[20] V. Kapaklis, U. B. Arnalds, A. Harman-Clarke, E. T. Papaioannou, M. Karimipour, P. Korelis, A. Taroni, P. C. W. Holdsworth, S. T. Bramwell, and B. Hjörvarsson, New J. Phys. 14, 035009 (2012).

[21] U. B. Arnalds, A. Farhan, R. V. Chopdekar, V. Kapaklis, A. Balan, E. T. Papaioannou, M. Ahlberg, F. Nolting, L. J. Heyderman, and B. Hjörvarsson, Appl. Phys. Lett. 101, 112404 (2012).

[22] A. Farhan, P. M. Derlet, A. Kleibert, A. Balan, R. V. Chopdekar, M. Wyss, L. Anghinolfi, F. Nolting, and L. J. Heyderman, Nat. Phys. 9, 375 (2013).

[23] V. Kapaklis, U. B. Arnalds, A. Farhan, R. V. Chopdekar, A. Balan, A. Scholl, L. J. Heyderman, and B. Hjörvarsson, Nat. Nanotechnol. 9, 514 (2014).

[24] L. Anghinolfi, H. Luetkens, J. Perron, M. G. Flokstra, O. Sendetskyi, A. Suter, T. Prokscha, P. M. Derlet, S. L. Lee, and L. J. Heyderman, Nat. Commun. 6, 8278 (2015).

[25] M. S. Andersson, S. D. Pappas, H. Stopfel, E. Östman, A. Stein, P. Nordblad, R. Mathieu, B. Hjörvarsson, and V. Kapaklis, Sci. Rep. 6, 37097 (2016).

[26] H. Stopfel, E. Östman, I.-A. Chioar, D. Greving, U. B. Arnalds, T. P. A. Hase, A. Stein, B. Hjörvarsson, and V. Kapaklis, Phys. Rev. B 98, 014435 (2018), and the related supplementary material. 
[27] O. Sendetskyi, V. Scagnoli, N. Leo, L. Anghinolfi, A. Alberca, J. Lüning, U. Staub, P. M. Derlet, and L. J. Heyderman, Phys. Rev. B 99, 214430 (2019).

[28] M. Pohlit, G. Muscas, I.-A. Chioar, H. Stopfel, A. Ciuciulkaite, E. Östman, S. D. Pappas, A. Stein, B. Hjörvarsson, P. E. Jönsson, and V. Kapaklis, Phys. Rev. B 101, 134404 (2020).

[29] N. Leo, M. Pancaldi, S. Koraltan, P. V. González, C. Abert, C. Vogler, F. Slanovc, F. Bruckner, P. Heistracher, K. Hofhuis, M. Menniti, D. Suess, and P. Vavassori, New J. Phys. 23, 033024 (2021).

[30] P. Mellado, Phys. Rev. B 102, 214442 (2020).

[31] B. E. Skovdal, N. Strandqvist, H. Stopfel, M. Pohlit, T. Warnatz, S. D. Slöetjes, V. Kapaklis, and B. Hjörvarsson, Phys. Rev. B 104, 014434 (2021).

[32] E. Ising, Z. Phys. 31, 253 (1925).

[33] E. Östman, U. B. Arnalds, V. Kapaklis, A. Taroni, and B. Hjörvarsson, J. Phys.: Condens. Matter 30, 365301 (2018).

[34] M. Pärnaste, M. Marcellini, E. Holmström, N. Bock, J. Fransson, O. Eriksson, and B. Hjörvarsson, J. Phys.: Condens. Matter 19, 246213 (2007).
[35] U. B. Arnalds, M. Ahlberg, M. S. Brewer, V. Kapaklis, E. T. Papaioannou, M. Karimipour, P. Korelis, A. Stein, S. Olafsson, T. P. A. Hase, and B. Hjörvarsson, Appl. Phys. Lett. 105, 042409 (2014).

[36] E. T. Papaioannou, V. Kapaklis, A. Taroni, M. Marcellini, and B. Hjörvarsson, J. Phys.: Condens. Matter 22, 236004 (2010).

[37] P. W. Anderson, Science 177, 393 (1972).

[38] K. G. Wilson, Sci. Am. 241, 158 (1979).

[39] S. Gliga, A. Kákay, R. Hertel, and O. G. Heinonen, Phys. Rev. Lett. 110, 117205 (2013).

[40] V. S. Bhat and D. Grundler, Phys. Rev. B 98, 174408 (2018).

[41] A. Ciuciulkaite, E. Östman, R. Brucas, A. Kumar, M. A. Verschuuren, P. Svedlindh, B. Hjörvarsson, and V. Kapaklis, Phys. Rev. B 99, 184415 (2019).

[42] P. Gypens, J. Leliaert, and B. Van Waeyenberge, Phys. Rev. Appl. 9, 034004 (2018).

[43] M. B. Jungfleisch, W. Zhang, E. Iacocca, J. Sklenar, J. Ding, W. Jiang, S. Zhang, J. E. Pearson, V. Novosad, J. B. Ketterson, O. Heinonen, and A. Hoffmann, Phys. Rev. B 93, 100401(R) (2016).

[44] S. Lendinez and M. B. Jungfleisch, J. Phys.: Condens. Matter 32, 013001 (2020). 